\title{
Musical Grammars as the Basis of Non-Speech Audio Communication
}

\author{
John C. K. Hankinson, Alistair D. N. Edwards \\ Department of Computer Science \\ University of York \\ York, England, YO10 5DD
}

\begin{abstract}
Non-speech audio communication has so far been possible through a series of mappings between the message to communicate and a somewhat arbitrary representation of that message in sound. By introducing the concept of a musical grammar, it is proposed that the arbitrariness of sound design can be reduced, resulting in a much more powerful communicative medium. By studying the ways in which formal grammars have been used to describe language communication and the parallels that such approaches have with musical grammars, it is shown that highly structured and communicatively-expressive sound systems which obey the rules of music can be developed, resulting in more advanced non-speech audio communication.
\end{abstract}

\section{Motivation}

This poster is concerned with the possibility of developing non-speech audio signals using grammatical descriptions of musical structures. It is envisaged that this approach to sound design will have a number of advantages over current techniques. First, using a grammar imposes structure and reduces the arbitrariness of sound design. At the same time, grammars can be very expressive, resulting in broad and powerful communication. Finally, using musical conventions means that users can rely on culturally-acquired musical knowledge.

\section{Objects and Actions}

To illustrate the grammatical approach, a simple object/action interface has been chosen. The objects consist of a number of files, printers, disks and texts. The set of accompanying actions include actions such as pause, bold, copy and print; these too could be grouped into a small number of categories. Only actions from a subset of the categories can act upon objects from a particular object category. In other words, each action category is associated with a number of object categories. These relationships can be captured within a grammar:

$$
\begin{array}{ll}
I \rightarrow \text { A2. Disks } \mid \text { A5. Disks } & \text { I } \rightarrow \text { A2. Files } \mid \text { A3. Files } \mid \text { A4 . Files } \\
\text { I } \rightarrow \text { A1. Printers } \mid \text { A3 . Printers } & \text { I } \rightarrow \text { A2. Texts } \mid \text { A6. Texts }
\end{array}
$$

where $\mathrm{I}$ is a legal interaction. The action categories A1 to A6 consist of:
A1: Pause, Resume
A3: Print
A5: Scan, Format
A2: Delete, Copy
A4: Open
A6: Italic, Bold

\section{Audio Representations}

Assuming the objects and actions are to be represented in sound, it would be beneficial if we could maintain the grammatical associations between categories. That is, the combination of object / action sounds must sound 'correct' when they form a legal interaction. Inappropriate combinations should sound 'wrong'. Therefore, a set of sounds is needed whose members exhibit the same associations as found between object and action categories. This can be achieved 
by imposing different chordal structures upon earcons of different categories. Chords will combine successfully when the categories match.

\subsection{Tonal Harmony}

A chord is simply a collection of tones (notes of a particular pitch). Some tones are known to combine well; others less so. If the ratios between pitch frequencies are simple, the tones harmonise well. Taking this effect into account, a set of tones can be ordered by ratio, resulting in something close to the 'overtone series'. Tonal harmony is a musical discipline which is founded on the overtone series. This theory describes how tones combine to form particular chords and how those chords can be ordered over time to form a sequence.

The function of a tone in a chord can be discerned by looking at the relationship between a tone in a chord and the tonic of that chord. When two chords combine, this function changes due to the new tonic of the combined chord. It is this effect which allows a chord to combine well with some chords (for our purposes, grammatically) whilst forming 'awkward' tonal relationships with others.

\subsection{A Musical Grammar}

A set of chords has been found which can adequately capture the grammatical relationships required for each category (the musical version of the grammar is shown below). It is hoped that by imposing this choral structure upon the design of object/action earcons, resultant combined earcons will be intuitively recognisable as grammatically correct or incorrect.

$$
\begin{aligned}
& \mathrm{I} \rightarrow \mathrm{Gm} \text { (no 5th). Eb } \mid \mathrm{Gm}^{\mathrm{b5}} . \mathrm{Eb} \\
& \mathrm{I} \rightarrow \mathrm{Am}^{\mathrm{b} 5} . \mathrm{F} \mid \mathrm{Dm} \text { (no 5th). F } \\
& \mathrm{I} \rightarrow \mathrm{Gm} \text { (no 5th). Bb|Dm (no 5th). Bb } \mid \mathrm{Dm}^{\mathrm{b} 5} . \mathrm{B} b \\
& \mathrm{I} \rightarrow \mathrm{Gm} \text { (no 5th). C } \mid \mathrm{Em}^{\mathrm{b} 5} \text {. C }
\end{aligned}
$$

Of course, the perceived well-formedness of a chord depends upon the context in which it appears. Some chords are more acceptable when subsequent chords resolve them. Also, personal experience alters the bounds of what is acceptable and what is not. However, this does not create too many problems as users can learn to recognise where the chosen boundary between dissonance and consonance lies for the given example.

\section{Conclusions}

The approach outlined above is presently an idea which needs further development and evaluation. It is hoped that a number of user studies will be undertaken in the future, possibly with a direct comparison between grammatical and traditional approaches to earcon design. Furthermore, the technique may be extended to include chord sequences and the effects of rhythm and melody for more powerful, highly-structured design.

\section{References}

[1] Blattner M. M., Sumikawa D. A., Greenberg R. M.,"Earcons and Icons: Their Structure and Common Design Principles", Human Computer Interaction, Vol. 4, 1989

[2] Brewster, S. A., Wright, P. C., Edwards, A. D. N., "Experimentally Derived Guidelines for the Creation of Earcons”, in Adjunct Proceedings of HCI'95: People and Computers, Huddersfield: BCS, 1995

[3] Holtzman S. R., Digital Mantras: The Languages of Abstract and Virtual Worlds, MIT Press, 1996

[4] Lerdahl F., Jackendoff R., "Toward a Formal Theory of Tonal Music”, Journal of Music Theory, Vol. 21, No. 1, 1997

[5] Steedman M. J., “A Generative Grammar for Jazz Chord Sequences”, Music Perception, Vol. 2, No. 1, 1984

ICAD '98 